\title{
The Assessment of Disease Activity in Psoriatic Arthritis: MDA, VLDA, DAPSA, or Something Else?
}

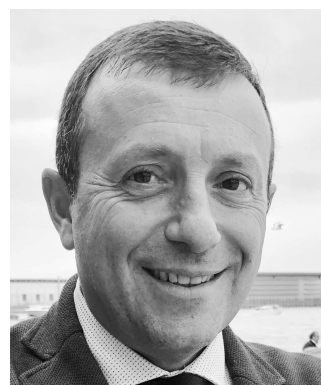

Psoriatic arthritis (PsA) is a multifaceted disease. Within this definition, rheumatologists can every day face various aspects of the disease and try to deal with them by choosing the right treatment. This could be a very easy task when some phenotypic manifestations and detectable, objective disease activity are present, but sometimes the same disease could be very challenging when some manifestations such as pain, fatigue, or even enthesitis are predominant and associated with less detectable disease activity.

Therefore, in the last decade, a significant effort has been made to identify potential instruments for the assessment of the disease activity as a "whole" and as the main target to be treated. In fact, rheumatologists have raised the bar, moving (as target to treat) from the achievement of good control of single domains such as pain, function, inflammation, skin, and quality of life to a more comprehensive disease control of PsA, aimed at managing the disease in all its components.

Indeed, the idea of developing a potential instrument to measure all disease domains was absolutely remarkable, and various ones were eventually developed and validated.

Minimal disease activity (MDA) ${ }^{1}$, very low disease activity (VLDA) ${ }^{2}$, and the Disease Activity Index for Psoriatic Arthritis (DAPSA) ${ }^{3}$ are those considered most useful for the assessment and they have been performed in posthoc analyses, real-world studies, and registries ${ }^{4}$.

In this issue of The Journal, Queiro, et al reported the results of a posthoc analysis of data from a cross-sectional multicenter study in Spain that aimed to examine the grade of agreement between VLDA and DAPSA ${ }^{5}$, as well as any potential association with the effect of the disease assessed by the Psoriatic Arthritis Impact of Disease (PsAID) in patients with $\mathrm{PsA}^{5,6}$. In this elegant and well-written paper, the authors concluded that VLDA seems to be more a stringent set of criteria for the identification of a remission state, in keeping with some other similar studies ${ }^{7,8,9}$, while DAPSA seems to be better correlated with PsAID, showing a better agreement between these 2 instruments.
Because the study was designed as a cross-sectional one and therefore with some potential limitation, the debate is whether VLDA status reflects a complete absence of disease activity and therefore an implicit condition of Patient Acceptable Symptom State (PASS), or whether DAPSA is the instrument to catch objective (musculoskeletal manifestations) and subjective (pain and patient's global assessment) components of the disease that patients perceived as important to judge themselves as in PASS. If we look at the PsA population studied in the paper by Queiro, et al, the peripheral joint involvement was predominant, so the achievement of a DAPSA remission was feasible, and potentially a PASS state by PsAID, too, if this instrument is more related to some aspect of disease caught by DAPSA. In fact, DAPSA, per se, is an effective unidimensional instrument for patients in which peripheral arthritis is the target to be treated, while MDA and VLDA are designed to go "deeper" to identify patients with PsA in low or very low disease activity by also evaluating other domains such as skin, enthesitis, or axial involvement. The latter, for instance, is a challenging subset of the disease and a few years ago we demonstrated that an MDA condition is achievable even in PsA patients with predominant axial PsA ${ }^{10}$.

Another aspect to consider from Queiro, et al is that the group receiving anti-tumor necrosis factor treatment in monotherapy was the one that achieved more of a VLDA status $^{5}$. Either that subgroup had milder conditions and consequently it was easier for them to achieve a status of remission, or alternatively, it was a "better responder" group. However, surprisingly, the patients receiving VLDA experienced less effect from the disease when measured by PsAID, even if the concordance was poor. The debate is still open and any ideas are well accepted but at present, data on a large number of patients would be welcome to sort out this intriguing result.

On the other side of the coin, the role of patients in the achievement of the treatment targets is fundamental and

See Remission in PsA, page 710

Personal non-commercial use only. The Journal of Rheumatology Copyright (C) 2019. All rights reserved. 
instruments such as PsAID are useful tools to be adopted in routine clinical practice, because of its capability to picture the global assessment of the disease from the patient's point of view. A proposal of different cutoffs corresponding to low disease activity by using PsAID in patients with PsA is currently in a validation study and is in keeping with the possibility of adopting this instrument to stratify the disease in various phases.

Having said that, PsAID is a reliable and valid instrument to assess the effect of PsA on patients' lives, and there is an increasing need to take on board the patient's assessment of their disease.

Therefore, is MDA, VLDA, or DAPSA the optimal instrument, or is it something else? There are different positions in the scientific community, but a strategy using PsAID in routine clinical practice could be useful. However, because of the heterogeneity of the disease, it could be possible that different domains, such as the cutaneous or musculoskeletal, are present in various moments and with different weights, influencing the final judgment of patients with PsA. Further, patients who achieved MDA or DAPSA remission have less radiographic progression of their disease, while this was still not demonstrated with PsAID ${ }^{11}$.

At present the optimal situation would be a multi-instrument assessment performed simultaneously in the clinic, or alternatively, just a patient's global assessment of the disease activity carried out using a visual analog scale, which we have shown to be an optimal surrogate of the disease activity ${ }^{12}$. Finally, patients with PsA attending an outpatient rheumatology clinic are mainly concerned with pain and articular complaints, and these are targets for the main treatment strategy to be chosen ${ }^{13}$.

\footnotetext{
ENNIO LUBRANO (D), MD, $\mathrm{PhD}$,

Associate Professor of Rheumatology,

Head of Academic Rheumatology Unit and MoRhe Project, Dipartimento di Medicina e Scienze della Salute, Università degli Studi del Molise,

Campobasso, Italy.
}

Address correspondence to Dr. E. Lubrano, University of Molise, Academic Rheumatology Unit, Department of Medicine and Health Sciences, Via Giovanni Paolo II, 86100 Campobasso, Italy.

E-mail: enniolubrano@hotmail.com

\section{REFERENCES}

1. Coates LC, Fransen J, Helliwell PS. Defining minimal disease activity in psoriatic arthritis: a proposed objective target for treatment. Ann Rheum Dis 2010;69:48-53.

2. Coates LC, Helliwell PS. Defining low disease activity states in psoriatic arthritis using novel composite disease instruments. J Rheumatol 2016;43:371-5.

3. Schoels MM, Aletaha D, Alasti F, Smolen JS. Disease activity in psoriatic arthritis (PsA): defining remission and treatment success using the DAPSA score. Ann Rheum Dis 2016;75:811-8.

4. Gossec L, McGonagle D, Korotaeva T, Lubrano E, de Miguel E, Ostergaard M, et al. Minimal disease activity as a treatment target in psoriatic arthritis: a review of the literature. J Rheumatol 2018; 45:6-13.

5. Queiro R, Canete JD, Montilla C, Angel Abad M, Montoro M, Gomez S, et al. Very low disease activity, DAPSA remission, and effect of disease in a Spanish population with psoriatic arthritis. J Rheumatol 2019;46:710-5.

6. Gossec L, de Wit M, Klitz U, Braun J, Kalyoncu U, Scrivo R, et al. A patient-derived and patient-reported outcome measure for assessing psoriatic arthritis: elaboration and preliminary validation of the Psoriatic Arthritis Impact of Disease (PsAID) questionnaire, a 13 country EULAR initiative. Ann Rheum Dis 2014;73:1012-9.

7. Lubrano E, Perrotta FM. Defining low disease activity states in psoriatic arthritis using novel composite disease instruments. J Rheumatol 2016;43:1765-6.

8. Lubrano E, De Socio A, Perrotta FM. Comparison of composite indices tailored for psoriatic arthritis treated with csDMARD and bDMARD: a cross-sectional analysis of a longitudinal cohort. J Rheumatol 2017;44:1159-64.

9. Coates LC, Lubrano E, Perrotta FM, Emery P, Conaghan PG, Helliwell PS. What should be the primary target of "treat to target" in psoriatic arthritis? J Rheumatol 2019 Sep 15 (E-pub ahead of print).

10. Lubrano E, Parsons WJ, Perrotta FM. Assessment of response to treatment, remission and minimal disease activity in axial psoriatic arthritis treated with tumor necrosis factor inhibitors. J Rheumatol 2016;43:918-23.

11. Helliwell PS, Kavanaugh A. Radiographic progression in psoriatic arthritis achieving a good response to treatment: data using newer composite indices of disease activity. Arthritis Care Res 2018;70:797-800.

12. Lubrano E, Perrotta FM, Parsons WJ, Marchesoni A. Patient's global assessment as an outcome measure for psoriatic arthritis in clinical practice: a surrogate for measuring low disease activity? J Rheumatol 2015;42:2332-8.

13. Lubrano E, Perrotta FM. Psoriatic arthritis: is it time to treat-to-target or target to treat? Clin Rheumatol 2017;36:2633-35.

J Rheumatol 2019;46:663-4; doi:10.3899/jrheum.181313 